\title{
Preventing adverse events of
} chemotherapy by educating patients about the nocebo effect (RENNO study) - study protocol of a randomized controlled trial with gastrointestinal cancer patients

\author{
Julia Quidde ${ }^{1+}$, Yiqi Pan ${ }^{2+}$, Melanie Salm ${ }^{3}$, Armin Hendi ${ }^{1,2}$, Sven Nilsson ${ }^{1}$, Karin Oechsle ${ }^{1}$, Alexander Stein ${ }^{4}$
} and Yvonne Nestoriuc ${ }^{2^{*}}$

\begin{abstract}
Background: Patients undergoing chemotherapy are highly burdened by side effects. These may be caused by the pharmacodynamics of the drug or be driven by psychological factors such as negative expectations or pre-conditioning, which reflect nocebo effects. As such, negative pre-treatment expectations or prior experiences might exacerbate the burden of chemotherapy side effects. Educating patients about this nocebo effect has been put forward as a potential strategy to optimize patients' pre-treatment expectations. In this study, we evaluate whether a briefing about the nocebo effect is efficacious in reducing side effects.

Methods: In this exploratory study, a total number of $n=100$ outpatients with newly diagnosed gastrointestinal cancers are randomized 1:1 to an information session about the nocebo effect (nocebo-education) or an attention control group (ACG) with matching interaction time. Assessments take place before the intervention (T1 pre), post-intervention (T1 post), and 10 days (T2) and 12 weeks (T3) after the initial chemotherapy. The primary outcomes are the patient-rated number and intensity of side effects at 10-days and at 12-weeks follow-up. Secondary outcomes include coping with side effects, tendency to misattribute symptoms, compliance intention, attitude towards the chemotherapy, co-medication to treat side effects and the clinician-rated severity of toxicity. Further analyses are conducted to investigate whether a potential beneficial effect is mediated by a change of expectations before and after the intervention.
\end{abstract}

Discussion: Informing patients about the nocebo effect might be an innovative and feasible intervention to reduce the burden of side effects and strengthen patients' perceived control over adverse symptoms.

Trial registration: The trial is registered at the German Clinical Trials Register (ID: DRKS00009501; retrospectively registered on March 27, 2018). The first patient was enrolled on September 29, 2015.

Keywords: Side effects, Chemotherapy, Gastrointestinal cancer, Treatment expectations, Nocebo effect, Informed consent

\footnotetext{
* Correspondence: y.nestoriuc@uke.de

${ }^{\dagger} J$ ulia Quidde and Yiqi Pan contributed equally to this work.

${ }^{2}$ Department of Psychosomatic Medicine and Psychotherapy, University

Medical Center Hamburg-Eppendorf, Martinistr. 52, 20246 Hamburg, Germany

Full list of author information is available at the end of the article
}

(c) The Author(s). 2018 Open Access This article is distributed under the terms of the Creative Commons Attribution 4.0 International License (http://creativecommons.org/licenses/by/4.0/), which permits unrestricted use, distribution, and reproduction in any medium, provided you give appropriate credit to the original author(s) and the source, provide a link to the Creative Commons license, and indicate if changes were made. The Creative Commons Public Domain Dedication waiver (http://creativecommons.org/publicdomain/zero/1.0/) applies to the data made available in this article, unless otherwise stated. 


\section{Background}

Gastrointestinal tumors, including cancer of the esophagus, stomach, colon, rectum, anus, pancreas, gallbladder and bile duct, constitute the largest group of cancer for men, and the second largest group of cancer for women [1]. Further, they are characterized by high mortality rates [1]. One of the treatment options for gastrointestinal tumors is chemotherapy, which can be indicated in the neoadjuvant, adjuvant or palliative setting [2]. Due to the high toxicity of the chemotherapy, cancer patients are commonly burdened by side effects of varying severity which may impair quality-of-life $[3,4]$. Furthermore, side effects have substantial weight in the individual decision process of whether or not to proceed with chemotherapy and were shown to be one of the three major predictors of treatment discontinuation in the palliative setting $[5,6]$. Strikingly, side effects are not fully defined by the pharmacodynamics of a given drug, but can also be caused by psychological factors. These so-called nocebo effects are best known as the counterparts of placebo effects [7]. The nocebo effect can depend on a variety of factors, e.g. the patient-practitioner relationship, a patient's treatment expectations, or prior learning experiences [8]. Nocebo-driven side effects can occur as unspecific side effects, i.e. symptoms not explainable by the drug, or may also exacerbate specific side effects [9]. Studies which investigated adverse events after placebo intake $[10,11]$, as well as studies which used verbal suggestions to manipulate treatment expectations prior to medication intake, demonstrated the existence of nocebo effects [12-14].

One of the main underlying mechanisms for the development of nocebo effects is expectations. These are formed based on prior experiences (either specific to the treatment or in general, to medical procedures) [15], social learning [16], and the information received about the treatment [17].

In clinical practice, patients are briefed about the treatment including potential risks in the informed consent. Although a great achievement towards patient autonomy, standard informed consent procedures may facilitate negative expectations which can, in turn, cause nocebo effects [18]. As reported in a meta-analysis, negative pre-treatment expectations predict the occurrence of actual side effects [19]. The strongest associations between symptom expectation and symptom occurrence were found for pain, fatigue, and nausea [19]. Moreover, negative treatment expectations prior to chemotherapy are high. In a study by Hofman and colleagues [20], most of the cancer patients expected that they would develop severe or long-lasting side effects such as fatigue (90.6\%) and nausea (78\%). In summary, optimizing informed consent procedures may be an essential module in the prevention of nocebo effects during chemotherapy.
By acknowledging the ethical and legal necessity of informed consent on the one side and the importance to minimize risks on the other, Barsky and colleagues [21] suggested educating patients about the nocebo effect. When aware of the existence of expectation-induced nocebo effects, patients' risk of misattributing unspecific symptoms to the treatment may be lower and they may be less vigilant to bodily signals, which again, may decrease the number and intensity of symptoms [22]. First evidence has shown that informing participants about the nocebo effect led to a reduction of symptoms that were allegedly caused by wind turbines [23]. Despite the high relevance of preventing nocebo effects during routine chemotherapy treatments, no comparable research has yet been conducted in the clinical context.

The primary aim of this exploratory clinical trial is to investigate the effects of a nocebo-education on the patientrated number and intensity of side effects in gastrointestinal cancer patients undergoing chemotherapy. By suggesting that side effects are not thoroughly determined by the pharmacodynamics of the treatment, patients' perceived self-efficacy to control side effects may be increased, and the misattribution of ambiguous body signals to the treatment may be reduced. Additionally, the nocebo-education may also facilitate the development of positive expectations in general, which in turn, can improve health outcomes [24]. Thus, we will investigate, as secondary outcomes, patients' perceived coping with side effects, tendency to misattribute symptoms, compliance intention, attitude towards the chemotherapy, clinician-rated severity of toxicity and type of co-medication used to treat side effects. Furthermore, we will examine whether optimized treatment expectations mediate the hypothesized beneficial effects of the nocebo-education. Since monitoring information coping styles have been associated with a higher report of side effects [25], we will investigate treatment information seeking as a moderator.

\section{Methods \\ Study design}

The RENNO trial (German Acronym for "Reduzierung des subjektiven Erlebens von Nebenwirkungen während der Chemotherapie durch Aufklärung über den Nocebo-Effekt") is a randomized-controlled, single-site superiority trial with two parallel arms and a primary endpoint of patient-reported number and intensity of side effects at 10 days and 12 weeks after the first chemotherapy session. Patients with newly diagnosed gastrointestinal cancers scheduled for chemotherapy are block-randomized 1:1 to a nocebo-education group or an attention control group entailing a quality-of-life interview.

The intervention takes place $<24 \mathrm{~h}$ before chemotherapy commences or during the administration of the first dose. Measurements are taken before (T1-pre), and after 
the intervention (T1-post), and 10 days (T2) and 12 weeks (T3) after the initial chemotherapy (Table 1).

\section{Trial registration and ethical approval}

Ethics approval has been given by the local ethics committee of the University of Hamburg. The trial has been retrospectively registered on March 27th, 2018 at the German Clinical Trial Register (ID: DRKS00009501).

\section{Study sample}

Patients with newly diagnosed cancer of the gastrointestinal tract, including the esophagus, stomach, colon, rectum, anus, pancreas, gallbladder and bile duct who are indicated for chemotherapy are eligible for this study. Chemotherapy can be given in the adjuvant, neoadjuvant, or first-line palliative setting, and is administered as a mono chemotherapy or combination-chemotherapy, with or without vascular endothelial growth factor (VEGF)-antibody. To enroll, patients must be at least 18 years old, have no difficulties understanding German, and be able to give informed consent. Exclusion criteria include the following: any chemotherapy before trial inclusion, limited capability of self-care (Eastern Co-operative Oncology Group $($ ECOG $\geq 3[26]$ ), severe comorbid psychological

Table 1 Schedule for enrolment, interventions and assessments

\begin{tabular}{|c|c|c|c|c|c|}
\hline \multirow[t]{3}{*}{ Time points } & \multicolumn{5}{|c|}{ Study period } \\
\hline & \multirow{2}{*}{$\begin{array}{l}\text { Allocation } \\
t_{1 \text { pre }}\end{array}$} & \multicolumn{4}{|c|}{ Post-allocation } \\
\hline & & $t_{1}$ & $t_{1 \text { post }}$ & $t_{2}$ & $t_{3}$ \\
\hline \multicolumn{6}{|l|}{ ENROLMENT } \\
\hline Eligibility screen ${ }^{a}$ & $\checkmark$ & & & & \\
\hline Informed consent & $\checkmark$ & & & & \\
\hline Randomized assignment & $\checkmark$ & & & & \\
\hline \multicolumn{6}{|l|}{ INTERVENTIONS } \\
\hline Nocebo-education group & & $\checkmark$ & & & \\
\hline Attention control group & & $\checkmark$ & & & \\
\hline \multicolumn{6}{|l|}{ ASSESSMENTS } \\
\hline \multicolumn{6}{|l|}{ Baseline variables } \\
\hline Demographic and medical characteristics & $\checkmark$ & & & & \\
\hline Distress Thermometer & $\checkmark$ & & & & \\
\hline Information coping style & $\checkmark$ & & $\checkmark$ & & \\
\hline \multicolumn{6}{|l|}{ Outcome variables } \\
\hline Side effect number and intensity (GASE) ${ }^{b}$ & & & & $\checkmark$ & $\checkmark$ \\
\hline Coping with side effects (GASE-Coping) & & & & $\checkmark$ & $\checkmark$ \\
\hline Misattribution of symptoms (GASE-Attribution) & & & & $\checkmark$ & $\checkmark$ \\
\hline Co-medication to treat side effects (yes/no) & & & & $\checkmark$ & $\checkmark$ \\
\hline Compliance intention & $\checkmark$ & & $\checkmark$ & $\checkmark$ & $\checkmark$ \\
\hline Attitude towards the chemotherapy & $\checkmark$ & & $\checkmark$ & $\checkmark$ & $\checkmark$ \\
\hline Severity of toxicity $(\mathrm{CTC})^{c}$ & $\checkmark$ & & & $\checkmark$ & $\checkmark$ \\
\hline \multicolumn{6}{|l|}{ Further variables } \\
\hline Expected side effects (GASE-Expect) & $\checkmark$ & & $\checkmark$ & & \\
\hline Expected coping with side effects (GASE-Coping Expect) & $\checkmark$ & & $\checkmark$ & & \\
\hline Expected efficacy of chemotherapy & & & $\checkmark$ & & \\
\hline Relevance of the conversation & & & $\checkmark$ & & \\
\hline Knowledge about the nocebo effect ${ }^{d}$ & & & $\checkmark$ & $\checkmark$ & $\checkmark$ \\
\hline Disease progression & & & & $\checkmark$ & $\checkmark$ \\
\hline
\end{tabular}

Note. The time points T1-pre and T1-post refer to the intervention whereas the T2 and T3 refer to the chemotherapy (10 days and 12 weeks after the initial chemotherapy). GASE Generic Assessment of Side Effects, CTC Common Toxicity Criteria

${ }^{a}$ The eligibility screening takes place in two steps. In the first step, medical eligibility criteria are checked, whereas in the second step, all further criteria are assessed as part of the health professional-patient communication right before the signing of the informed consent

${ }^{\mathrm{b}}$ Assessed symptoms include fatigue, nausea, diarrhea, vomiting, headache, shortness of breath, and rash

'Severity of toxicity is assessed by the clinician at every cycle

${ }^{d}$ Knowledge about the nocebo effect is only assesses in the nocebo education group 
disorder (schizophrenia, substance abuse, severe depression or severe anxiety disorder), concurrent psychotherapeutic or psychopharmacological treatments, life-threatening comorbid medical condition, diagnosis of chronic skin disease, dyspnea, or chronic lung diseases due to its interference with chemotherapy side effects, indication for epidermal growth factor receptor (EGFR)-antibody treatment since it may cause acne-like rash. Since prior research has found that the link between negative treatment expectations and side effects was more pronounced among patients who had chemotherapy experience [19], we excluded patients with prior chemotherapy experience, including having received the first dose before enrolment, to achieve an increased homogeneity of the sample.

\section{Recruitment}

Patients are recruited from the University Medical Center (UMC) Hamburg-Eppendorf, Department of Oncology, Hematology and Bone Marrow Transplantation and from cooperating practices. All patients are treated in an outpatient setting. With the aim to achieve adequate enrolment of participants, each patient with a newly diagnosed gastrointestinal cancer treated at the Department of Oncology of the UMC is screened. The first screening of inclusion criteria is conducted by the patient's treating oncologist.

The healthcare professional (medical doctoral candidate or Psychologist, B.Sc.) informs patients about the study's aim ("gaining an enhanced understanding of the quality-of-life of patients which undergo chemotherapy including potential worries and expectations about the treatment") and procedures ("assignment to one of two possible conversations"). Further eligibility criteria are assessed through the healthcare professional by inquiring the patient directly (e.g. ECOG score). Informed consent is obtained by the same healthcare professional prior to enrolment.

\section{Randomization and blinding}

A stratified block randomization is performed after the signing of the informed consent. To prevent a potential selection bias due to predictability, the block sizes are not noted here. The stratum distress is assessed at T1-pre using a distress-thermometer, a commonly used screening instrument among oncological patients ranging from 0 (not distressed) to 10 (extremely distressed) [27]. The allocation sequence for each stratum category (distress $<5$ 'low' vs. distress $\geq 5$ 'high') is produced by a research assistant prior enrolment using a computer program [28]. Sequentially numbered, opaque, sealed envelopes are used for the concealment of group assignment. Randomization is performed by a trained healthcare professional who, according to the distress level indicated by the patient, draws the envelope from the respective box. Patients are present during the allocation and are informed about their assignment instantaneously.

Due to the nature of the study, patients are not blinded. However, they are unaware of the specific study hypotheses and the content of the intervention that they do not receive. The trained healthcare professional is not blinded to group assignment. However, we aimed to confine the post-randomization interaction between the trained healthcare professional and the patient to the intervention. Follow-up assessments are conducted independently via mail using a standardized cover letter, i.e., no further interactions take place. Only in cases when patients do not complete assessment, the healthcare professional conducts a reminder call which is manualized in advance.

\section{Interventions}

Both interventions take place right after the randomization and are carried out by trained healthcare professionals in an empathetic, patient-centered manner and are of similar duration (15-20 min). The attention control group controls for unspecific effects like attention by the healthcare professional or a positive patient-practitioner relationship. The intervention is conducted in addition to clinical routine and is scheduled individually between the healthcare professional and the patient to achieve the best fit in line with the patient's schedule. Pre-defined as exclusion criteria, concomitant psychotherapy or psychopharmacological treatments are prohibited. Also as part of the clinical routine, all patients receive prescriptions for medications against nausea/vomiting and diarrhea.

\section{Nocebo-education group}

The nocebo-education is a manualized information session with the objective of illustrating the nocebo effect in a comprehensive way using patient-oriented language and practical examples [18]. It was developed as part of an experimental pilot-study by the research team of the PI of this study (YN) and has been adapted according to patient feedback. The intervention includes the following five steps: (1) The healthcare professional asks the patient about their personal experiences with side effects of medications or other treatments. (2) A case example of a nocebo response in the clinical context is introduced and discussed (adapted from [29]). (3) A standardized leaflet about the nocebo effect is handed out (see Table 2) and discussed. Patients are invited to talk about their own thoughts and potential prior experiences with nocebo responses. (4) Lastly, patients are encouraged to apply the obtained information to their imminent chemotherapeutic experience. The manual accounts for potential deviations of patients' experiences and recall abilities (e.g. if the patient cannot recall any 
Table 2 Brief discussion points from the leaflet of the nocebo-education

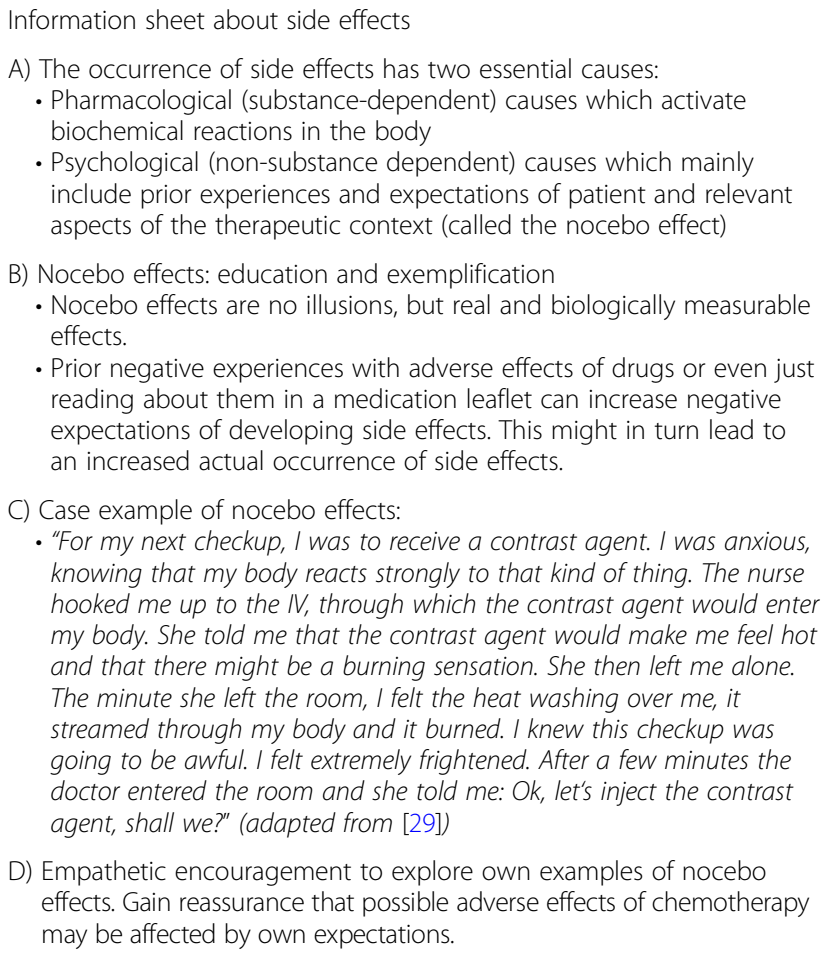

B) Nocebo effects: education and exemplification

- Nocebo effects are no illusions, but real and biologically measurable effects.

- Prior negative experiences with adverse effects of drugs or even just reading about them in a medication leaflet can increase negative expectations of developing side effects. This might in turn lead to an increased actual occurrence of side effects.

C) Case example of nocebo effects:

- "For my next checkup, I was to receive a contrast agent. I was anxious, knowing that my body reacts strongly to that kind of thing. The nurse hooked me up to the $N$, through which the contrast agent would enter my body. She told me that the contrast agent would make me feel hot and that there might be a burning sensation. She then left me alone. The minute she left the room, I felt the heat washing over me, it streamed through my body and it burned. I knew this checkup was going to be awful. I felt extremely frightened. After a few minutes the doctor entered the room and she told me: Ok, let's inject the contrast agent, shall we?" (adapted from [29])

D) Empathetic encouragement to explore own examples of nocebo effects. Gain reassurance that possible adverse effects of chemotherapy may be affected by own expectations.

individual nocebo responses, the health professional may use a personal example).

\section{Attention control group}

A face valid topic of matching relevance to the research topic of this trial is discussed with the patients in the attention control group in a similarly manualized manner. Based on the Functional Assessment of Cancer Therapy general scale (FACT-G; [30]), the healthcare professional makes inquiries about the quality-of-life subthemes physical well-being, emotional well-being and functional well-being using a semi-structured interview format. The two themes beliefs and spirituality, and relationship to practitioners which are not part of the scale are additionally addressed. Twenty questions are asked in total. The healthcare professional or the patient may address issues in further depth. Some items are rephrased to achieve a balance of negatively and positively phrased items (e.g. "I am worried that my condition will worsen" is rephrased as "I am optimistic about the future"). Answers are noted on the questionnaire. Answers to the interview are not part of the research questions and are hence not analyzed.

\section{Data collection and confidentiality}

Self-report data is collected at before and after the intervention (T1-pre and T1-post) by the healthcare practitioner. Further self-report data at 10 days and 12 weeks follow-up is collected via mail. Medical characteristics are assessed using medical records. Clinician-rated data are collected at every chemotherapy cycle. All data in paper form will be stored at the UMC Hamburg-Eppendorf in rooms inaccessible to non-research staff. Since we conduct repeated measurements, identifying information of the patient is substituted by a code. Patients' personal information (name, phone number, address) are securely stored separately from data which are outlined under "measures".

\section{Measures \\ Primary outcomes}

The mean group difference in number and intensity of patient-reported side effects at 10 days and 12 weeks after the initial chemotherapy session are defined as primary outcomes. Based on the Generic Assessment of Side Effects (GASE) [31], we chose four side effects specific to the chemotherapy, i.e., nausea, vomiting, diarrhea, and fatigue [32, 33]. Three unspecific symptoms, i.e., headache, shortness of breath, and rash, were defined as side effects that were very unlikely to occur in pharmacodynamic relation to the chemotherapy. They were chosen as a result of discussions among the research staff oncologists. A global item assesses the overall intensity of side effects in the last 7 days. The original GASE ranged from 0 to 3; to optimize outcome sensitivity in this exploratory trial, we increased the item range from 0 (not at all) to 10 (very much). Numbers of side effects are computed by adding up the number of present symptoms $(\geq 1)$.

\section{Secondary outcomes}

Coping with side effects, the tendency to misattribute symptoms, compliance intention, attitude towards the chemotherapy, co-medication to treat side effects, and clinician-rated severity of toxicity measured at 10 days and 12 weeks after the initial chemotherapy session are examined as secondary outcomes. Coping with side effects is assessed as part of the GASE (GASE-Coping; varied after [34]). For each side effect, patients are asked to indicate how well they felt able to cope with the symptom (0 'not at all' to 10 'very much'). Accordingly, the tendency to attribute symptoms ("To which degree do you attribute [symptom] to the chemotherapy?") is assessed for each side effect on the same scale. The tendency to misattribute is computed as the mean attribution tendency of unspecific symptoms to the chemotherapy. Compliance intention to chemotherapy is assessed with two items ("How sure are you that you will complete your chemotherapy cycle?" from 0 "not at all" to 10 "very much"; "How high is the probability that you will discontinue your chemotherapy?" from 0 to $100 \%)$. Attitude towards the chemotherapy is measured using one item 
("How would you describe your attitude towards the chemotherapy") which ranges from 0 (very negative) to 10 (very positive). Co-medication to treat side effects is assessed with a yes/no question. The clinician-rated severity of toxicity is assessed using the Common Toxicity Criteria (CTC) Version 4.03 at every cycle. The CTC provides standardized definitions of adverse events ranging from grade 1 to grade 5 with each grade reflecting a distinct description of the adverse events severity [35].

\section{Mediating variables}

Expected side effect intensity and expected coping with side effects are assessed with a modified version of the GASE [34] before (T1-pre) and after the intervention (T1-post). Patients indicate the expected intensity and coping for each of the 7 symptoms (0 'not at all' to 10 'very much').

\section{Baseline variables}

Gender, age, education, employment, psychological distress [27], the affected gastrointestinal area, physical and psychological co-morbidities are assessed using self-report. Tumor staging, aim of treatment (neoadjuvant, adjuvant, palliative etc.), and the chemotherapeutic regime are obtained from medical records. Information coping styles are assessed with two items ("To which degree do you wish to be informed about the chemotherapy?", "How strongly do you feel the need to inform yourself about potential side effects?"), both rated on a scale of 0 (not at all) to 10 (very much).

\section{Manipulation check, evaluation of the nocebo-education, process variables}

Comprehension of the nocebo effect information is reviewed by asking patients in the nocebo group to describe the nocebo effect. Perceived relevance of the nocebo-education and the control intervention is assessed at T1-post. Expected efficacy of chemotherapy includes four items that assess to which degree the patient anticipates the treatment will 1) help them recover, 2) shrink the tumor, 3) improve their quality-of-life and 4) prolong their life. Each item ranges from 0 (not at all) to 10 (very much). Progression of cancer is assessed from medical records during the study period.

\section{Sample size calculation}

In a pilot study which aimed to validate the nocebo-education session, we found a moderate to large effect (Cohen's $d=0.50-0.65$ ) when comparing post-session side effect expectations between participants who were educated vs. those who were not educated. Since no prior study has been conducted to examine the effect of a nocebo education on side effects, we used the aforementioned effect size of side effect expectations as a proxy for actual side effects. Hence, when conducting an independent $t$-test, given an effect size of $d=0.60$, the desired power of $80 \%$, and an alpha-error of 0.05 (two-tailed testing), we obtained a required sample size of $N=45$ per group for this exploratory clinical study. When accounting for a dropout rate of $10 \%$, a total sample of $N=100$ is needed to investigate potential group differences with adequate power for our primary outcome burden of side effects.

\section{Data management}

After data entry, $10 \%$ of the electronic data is checked for correspondence with the original paper data. Plausibility checks (e.g. for item range and scale ranges) are conducted.

\section{Statistical methods}

All outcome analyses will be conducted for the intention-to-treat sample. Missing values will be imputed using the expectation-maximization algorithm. We will conduct ANCOVA for the primary outcomes number and intensity of side effects, and for the secondary outcomes coping with side effects, tendency to misattribute symptoms, compliance intention, attitude towards chemotherapy, and medical toxicity at both time points (10 days and 12 weeks) with adjustments for the stratum distress and for cancer staging. Logistic regression will be conducted for the secondary outcome co-medication to treat side effects with the same set of covariates. The T1-pre to T1-post changes in expected side effects and expected coping with side effects will be examined as mediators of the effect of group on the outcomes. Exploratory analyses will be conducted to compare the expected efficacy of the chemotherapy between groups using independent t-tests.

Moderate analyses will be carried out by including the group $\mathrm{x}$ information coping style interaction in the ANCOVA. Sensitivity analyses will be conducted under exclusion of (1) patients of the nocebo-education group who either indicated to not have heard about the nocebo effect or who gave a wrong description and (2) patients with tumor progression.

No interim analyses will be conducted.

\section{Discussion}

This is the first study to evaluate whether a nocebo-education can have beneficial effects considering side effects of the chemotherapy when compared to an attention control group. Up to now, no study has evaluated whether informing patients about the nocebo effect would be useful to optimize expectations and decrease side effect load in clinical practice. If efficacious, this trial would contribute to solving the ethical dilemma posed by the necessity and importance of informing the 
patients about the treatment on the one side and the potential negative effects of the information on the development of side effects on the other. Our objective was to create a nocebo-information session which is short, patient-oriented, and feasible in regard to implementation into clinical routine. Also, since the nocebo-education can be provided additional to the practitioner's information, no further changes to the clinical practice are necessary.

The following limitations should be noted: As the first exploratory trial to test hypothesized effects of a nocebo-education, the sample size in this study is rather small. Also, no specific power calculation was possible since the effects of a nocebo-education on side effects are unknown. Given the link between side effect expectations and side effects, we based our power calculations on the effects of a nocebo-education on side effect expectations. Nonetheless, the use of a proxy outcome for the sample size calculation constitutes a limitation. Due to the nature of the study, blinding of patients and healthcare professional was not possible. Whereas the healthcare professional is the assessor at T1-pre and T1-post, outcome assessments at 10 days and 12 weeks after the initial chemotherapy are conducted via mail to lessen potential biases through the assessor. Since our patient population is expected to be highly burdened, we aim to keep the additional effort which may arise through questionnaire completion to a minimum. Hence, except for our primary outcomes, we use several one-item or two-item questionnaires which have not been previously validated. Lastly, our primary outcome is based on patient self-report. If this trial shows beneficial effects of the nocebo-education, further studies including physiological measures or clinician-rated measures as primary outcomes would be valuable.

Preventing side effects of chemotherapy is essential. For example, fatigue alone can occur for months during the treatment, and for $20-34 \%$ of patients, persists up to 5 years after treatment completion $[36,37]$, perennially affecting quality-of-life $[20,38]$. Side effects of chemotherapy are also linked to increased anxiety, insecurity and a feeling of loss of control [39]. Hence, the development of innovative interventions to reduce side effects can be beneficial for the patient in the short and in the long run, as well as for public health systems.

\footnotetext{
Abbreviations

ACG: Attention control group; CTC: Common Toxicity Criteria; ECOG: Eastern Cooperative Oncology Group; EGFR: Epidermal growth factor receptor; FACTG: Functional Assessment of Cancer Therapy-General scale; GASE: Generic Assessment of Side Effects; RENNO: Reducing subjectively perceived side effects of the chemotherapy by educating about the nocebo effect [Reduzierung des subjektiven Erlebens von Nebenwirkungen während der Chemotherapie durch Aufklärung über den Nocebo-Effekt]; VEGF: Vascular endothelial cell growth factor
}

\section{Acknowledgments}

The authors thank Twyla Michnevich for proofreading the English manuscript.

\section{Funding}

The study is founded by the Foundation for the Science of the Therapeutic Encounter (F-STE). The funders played no role in the design of the study, and will play no role in the collection, analysis, and interpretation of data or in the decision to submit the manuscript for publication.

\section{Authors' contributions}

YN is the PI of the study. YN, JQ, YP, KO and AS initiated the study design. $\mathrm{MS}$ and $\mathrm{AH}$ recruit patients and conduct the interventions. JQ, AS, KO and SN provided oncological expertise and help with implementation. YP, JQ and YN drafted the manuscript. All authors made refinements to the protocol and approved the final manuscript.

\section{Ethics approval and consent to participate}

Ethics approval to the trial protocol version 1.0 (Hamburg, June 16, 2015) has been given by the local ethics committee of the University of Hamburg (Reference number: 2015_03). Any amendments to the protocol will be communicated and re-approved by the ethics committee. All participants will be informed about the study in verbal and written form before the consent procedure. Informed consent is obtained from all participants prior enrolment.

\section{Consent for publication}

Not applicable

\section{Competing interests}

The authors declare that they have no competing interests.

\section{Publisher's Note}

Springer Nature remains neutral with regard to jurisdictional claims in published maps and institutional affiliations.

\section{Author details}

${ }^{1}$ Department of Oncology, Haematology, Bone Marrow Transplantation with Section Pneumology, University Medical Center Hamburg-Eppendorf, Martinistr. 52, 20246 Hamburg, Germany. 'Department of Psychosomatic Medicine and Psychotherapy, University Medical Center Hamburg-Eppendorf, Martinistr. 52, 20246 Hamburg, Germany. ${ }^{3}$ Clinical Psychology and

Psychotherapy, University of Hamburg, Von-Melle-Park 5, 20146 Hamburg, Germany. ${ }^{4}$ University Cancer Center Hamburg, Hubertus Wald Tumorzentrum, University Hospital Hamburg-Eppendorf, Martinistr. 52, 20246 Hamburg, Germany.

Received: 14 June 2018 Accepted: 12 September 2018

Published online: 24 September 2018

\section{References}

1. Robert Koch-Institute: Krebs in Deutschland für 2013/2014. Edited by Zentrum für Krebsregisterdaten und der Gesellschaft der epidemiologischen Krebsregister in Deutschland e.V.; 2017.

2. DeSantis $C E$, Lin $C C$, Mariotto $A B$, Siegel RL, Stein $K D$, Kramer JL, Alteri $R$, Robbins AS, Jemal A. Cancer treatment and survivorship statistics, 2014. CA Cancer J Clin. 2014;64(4):252-71.

3. Wagland R, Richardson A, Ewings S, Armes J, Lennan E, Hankins M, Griffiths P. Prevalence of cancer chemotherapy-related problems, their relation to healthrelated quality of life and associated supportive care: a cross-sectional survey. Support Care Cancer. 2016;24(12):4901-11.

4. Cohen L, de Moor CA, Eisenberg P, Ming EE, Hu H. Chemotherapy-induced nausea and vomiting-incidence and impact on patient quality of life at community oncology settings. Support Care Cancer. 2007;15(5):497-503.

5. Clarke G, Johnston S, Corrie P, Kuhn I, Barclay S. Withdrawal of anticancer therapy in advanced disease: a systematic literature review. BMC Cancer. 2015:15:892.

6. Kuchuk I, Bouganim N, Beusterien K, Grinspan J, Vandermeer L, Gertler S, Dent SF, Song X, Segal R, Mazzarello S, et al. Preference weights for chemotherapy side effects from the perspective of women with breast cancer. Breast Cancer Res Treat 2013:142(1):101-7.

7. Kennedy WP. The nocebo reaction. Med World. 1961;95:203-5. 
8. Di Blasi Z, Harkness E, Ernst E, Georgiou A, Kleijnen J. Influence of context effects on health outcomes: a systematic review. Lancet. 2001;357(9258): 757-62

9. Colloca L, Finniss D. Nocebo effects, patient-clinician communication, and therapeutic outcomes. JAMA. 2012;307(6):567-8.

10. de la Cruz M, Hui D, Parsons HA, Bruera E. Placebo and nocebo effects in randomized double-blind clinical trials of agents for the therapy for fatigue in patients with advanced cancer. Cancer. 2010;116(3):766-74.

11. Petersen GL, Finnerup NB, Colloca L, Amanzio M, Price DD, Jensen TS, Vase L. The magnitude of nocebo effects in pain: a meta-analysis. Pain. 2014; 155(8):1426-34

12. Mondaini N, Gontero P, Giubilei G, Lombardi G, Cai T, Gavazzi A, Bartoletti R Finasteride $5 \mathrm{mg}$ and sexual side effects: how many of these are related to a nocebo phenomenon? J Sex Med. 2007;4(6):1708-12.

13. Varelmann D, Pancaro C, Cappiello EC, Camann WR. Nocebo-induced hyperalgesia during local anesthetic injection. Anesth Analg. 2010; 110(3):868-70

14. Bingel U, Wanigasekera V, Wiech $K$, Ni Mhuircheartaigh $R$, Lee MC, Ploner $M$, Tracey I. The effect of treatment expectation on drug efficacy: imaging the analgesic benefit of the opioid remifentanil. Sci Transl Med. 2011;3(70): 70 ra14.

15. Colloca L, Petrovic P, Wager TD, Ingvar M, Benedetti F. How the number of learning trials affects placebo and nocebo responses. Pain. 2010:151(2):430-9.

16. Benedetti F, Durando J, Vighetti S. Nocebo and placebo modulation of hypobaric hypoxia headache involves the cyclooxygenase-prostaglandins pathway. Pain. 2014;155(5):921-8.

17. Benedetti F, Lanotte M, Lopiano L, Colloca L. When words are painful: unraveling the mechanisms of the nocebo effect. Neuroscience. 2007; 147(2):260-71

18. Bingel U, Placebo Competence T. Avoiding nocebo effects to optimize treatment outcome. JAMA. 2014;312(7):693-4.

19. Sohl SJ, Schnur JB, Montgomery GH. A meta-analysis of the relationship between response expectancies and cancer treatment-related side effects. J Pain Symptom Manag. 2009;38(5):775-84.

20. Hofman M, Morrow GR, Roscoe JA, Hickok JT, Mustian KM, Moore DF, Wade $J$, Fitch TR. Cancer patients' expectations of experiencing treatment-related side effects: a University of Rochester Cancer Center--Community Clinical Oncology Program study of 938 patients from community practices. Cancer. 2004;101(4):851-7.

21. Barsky AJ, Saintfort R, Rogers MP, Borus JF. Nonspecific medication side effects and the nocebo phenomenon. JAMA. 2002;287(5):622-7.

22. Geers AL, Helfer SG, Weiland PE, Kosbab K. Expectations and placebo response: a laboratory investigation into the role of somatic focus. J Behav Med. 2006;29(2):171-8.

23. Crichton F, Petrie KJ. Health complaints and wind turbines: the efficacy of explaining the nocebo response to reduce symptom reporting. Environ Res. 2015;140:449-55.

24. Howick J, Moscrop A, Mebius A, Fanshawe TR, Lewith G, Bishop FL, Mistiaen $P$, Roberts NW, Dieninyte E, Hu XY, et al. Effects of empathic and positive communication in healthcare consultations: a systematic review and metaanalysis. J R Soc Med. 2018. https://doi.org/10.1177/0141076818769477.

25. Miller SM. Monitoring versus blunting styles of coping with Cancer influence the information patients want and need about their disease - implications for Cancer screening and management. Cancer. 1995;76(2):167-77.

26. Oken MM, Creech RH, Tormey DC, Horton J, Davis TE, McFadden ET, Carbone PP. Toxicity and response criteria of the eastern cooperative oncology group. Am J Clin Oncol. 1982;5(6):649-55.

27. Mehnert A, Müller D, Lehmann C, Koch U. Die deutsche Version des NCCN Distress-Thermometers: Empirische Prüfung eines Screening-Instruments zur Erfassung psychosozialer Belastung bei Krebspatienten. Z Psychiatr Psychol Psychother. 2006;54(3):213-23.

28. Abramson JH. WINPEPI (PEPI-for-windows): computer programs for epidemiologists. Epidemiol Perspect Innov. 2004;1(1):6.

29. Zimmermann T, Heinrichs N. Seite an Seite - eine Krebserkrankung in der Partnerschaft gemeinsam bewältigen. Ein Ratgeber für Paare. Göttingen: Hogrefe; 2008.

30. Cella DF, Tulsky DS, Gray G, Sarafian B, Linn E, Bonomi A, Silberman M, Yellen SB, Winicour P, Brannon J, et al. The functional assessment of Cancer therapy scale: development and validation of the general measure. J Clin Oncol. 1993:11(3):570-9.
31. Rief W, Barsky AJ, Glombiewski JA, Nestoriuc Y, Glaesmer H, Braehler E. Assessing general side effects in clinical trials: reference data from the general population. Pharmacoepidemiol Drug Saf. 2011;20(4):405-15.

32. Chen AB, Cronin A, Weeks JC, Chrischilles EA, Malin J, Hayman JA, Schrag D. Expectations about the effectiveness of radiation therapy among patients with incurable lung cancer. J Clin Oncol. 2013;31(21):2730-5.

33. Hochster HS, Hart LL, Ramanathan RK, Childs BH, Hainsworth JD, Cohn AL, Wong L, Fehrenbacher L, Abubakr Y, Saif MW, et al. Safety and efficacy of oxaliplatin and fluoropyrimidine regimens with or without bevacizumab as first-line treatment of metastatic colorectal cancer: results of the TREE study. J Clin Oncol. 2008;26(21):3523-9.

34. von Blanckenburg P, Schuricht F, Albert US, Rief W, Nestoriuc Y. Optimizing expectations to prevent side effects and enhance quality of life in breast cancer patients undergoing endocrine therapy: study protocol of a randomized controlled trial. BMC Cancer. 2013;13:426.

35. National Institutes of Health, National Cancer Institute: Common Terminology Criteria for Adverse Events (CTCAE). Version 4.03. In.; 2010.

36. Goldstein D, Bennett B, Friedlander M, Davenport T, Hickie I, Lloyd A. Fatigue states after cancer treatment occur both in association with, and independent of, mood disorder: a longitudinal study. BMC Cancer. 2006;6:240.

37. Bower JE, Ganz PA, Desmond KA, Bernaards C, Rowland JH, Meyerowitz BE, Belin TR. Fatigue in long-term breast carcinoma survivors: a longitudinal investigation. Cancer. 2006;106(4):751-8.

38. Curt GA, Breitbart W, Cella D, Groopman JE, Horning SJ, Itri LM, Johnson DH, Miaskowski C, Scherr SL, Portenoy RK, et al. Impact of cancer-related fatigue on the lives of patients: new findings from the fatigue coalition. Oncologist. 2000:5(5):353-60.

39. Pedersen B, Koktved DP, Nielsen LL. Living with side effects from cancer treatment--a challenge to target information. Scand J Caring Sci. 2013;27(3): $715-23$

Ready to submit your research? Choose BMC and benefit from:

- fast, convenient online submission

- thorough peer review by experienced researchers in your field

- rapid publication on acceptance

- support for research data, including large and complex data types

- gold Open Access which fosters wider collaboration and increased citations

- maximum visibility for your research: over $100 \mathrm{M}$ website views per year

At BMC, research is always in progress.

Learn more biomedcentral.com/submissions 